\title{
A Dynamic State-Space Model of Coded Political Texts
}

\author{
Martin Elff \\ Fachbereich Politik- und Verwaltungswissenschaft, Universität Konstanz, Universitätsstraße 10, \\ D-78464, Konstanz, Germany \\ e-mail: martin.elff@uni-konstanz.de (corresponding author) \\ Edited by Jonathan Katz
}

\begin{abstract}
This article presents a new method of reconstructing actors' political positions from coded political texts. It is based on a model that combines a dynamic perspective on actors' political positions with a probabilistic account of how these positions are translated into emphases of policy topics in political texts. In the article it is shown how model parameters can be estimated based on a maximum marginal likelihood principle and how political actors' positions can be reconstructed using empirical Bayes techniques. For this purpose, a Monte Carlo Expectation Maximization algorithm is used that employs independent sample techniques with automatic Monte Carlo sample size adjustment. An example application is given by estimating a model of an economic policy space and a noneconomic policy space based on the data from the Comparative Manifesto Project. Parties' positions in policy spaces reconstructed using these models are made publicly available for download.
\end{abstract}

\section{Introduction}

The study of spatial models of party competition and voting has made progress in the past decades from a purely formal exercise (e.g., McKelvey 1979; Enelow and Hinich 1984, 1990) to a subject of empirical investigation (e.g., Ezrow 2007; Adams and Merrill 2009), thanks to the availability of coded political texts, especially due to the availability of coded electoral platforms compiled and published by the Comparative Manifesto Project (CMP) (Budge, Robertson, and Hearl 1987; Budge et al. 2001; Klingemann et al. 2006; Volkens et al. 2010).

Data sets based on coded political texts generally consist of percentages or frequency counts of words, sentences, or quasi-sentences. Often, these frequency counts refer to broader coding categories that are assumed to have a particular political meaning, as in the case of the CMP data set, whereas in other cases the counts refer to words (Laver, Benoit, and Garry 2003; Slapin and Proksch 2008). When such data sets are used, it is generally assumed that the frequency of occurrences of particular words or broader categories of sentences are indicators of political actors' positions, because the actors presumably express their positions by using certain words more often or refer to certain political topics more often than others. But it is often overlooked - and this applies especially to studies that make use of the now popular left-right index constructed by the CMP scholars - that positions in a political space are conceptually different from frequency counts of words or references to political topics. A similar conceptual difference exists and has been acknowledged in the field of cognitive testing (McDonald 1999) or in the field of reconstructing legislators' ideal points from their votes recorded in a roll call (Poole and Rosenthal 1985; Martin and Quinn 2002; Clinton, Jackman, and Rivers 2004). The main implication of this conceptual

Author's note: I wish to thank Ken Benoit, Ian Budge, Daniel Stegmüller, Shawn Treier, Paul Whiteley, participants at the 2008 Summer Political Methodology meeting and the 2012 MPSA General Conference, the Departmental Seminars of the Department of Government and the Department of Mathematical Sciences of the University of Essex, and of the Research Seminar at Nuffield College, University of Oxford, as well as two anonymous reviewers for helpful comments and suggestions on previous versions of the article. Replication material, software, and supplementary data are available online at the Political Analysis dataverse. An online appendix on further details is available on the Political Analysis Web site.

(C) The Author 2013. Published by Oxford University Press on behalf of the Society for Political Methodology.

All rights reserved. For Permissions, please email: journals.permissions@oup.com 
difference is that positions in a political space cannot just be identified with a linear function (Budge, Robertson, and Hearl 1987; Budge et al. 2001; Klingemann et al. 2006; Volkens et al. 2010), and not even with a nonlinear function (Lowe et al. 2011), of the frequency counts or percentages of words or political categories in a text, just as a linear or nonlinear function of votes in a roll call cannot just be identified with the legislators' ideal points themselves.

The existence of the conceptual differences between frequency counts (which function as indicators) and actors' positions (which play the role of values of a latent variable) calls for a measurement model that is able to bridge this gap. While it may happen that some linear or nonlinear functions of sums of counts are better indicators of actors' political positions than others, one needs such a measurement model in order to adjudicate between different indicators, in the same way as Item Response Theory (IRT) models are used to examine and assess the validity of cognitive tests (McDonald 1999). ${ }^{1}$

The present article proposes such a model. Yet, it not only specifies a relation between the positions of political actors and observed data in the form of frequency counts of categories used in coding political texts, but it also has a dynamic component. Such a dynamic component is especially useful if one has political texts by political actors from more than just a few points in time, e.g., from only a few elections. This dynamic component allows us not only to describe the long-term development of political positions, but also to make use of the temporal succession of political texts for the reconstruction of actors' positions, instead of reconstructing them at each point in time in isolation.

In addition to specifying a measurement model, this article also presents a method of estimating political positions motivated by it. It is further demonstrated how this estimation method can be applied to the CMP data or other bodies of coded political texts and how to test statistical hypotheses about the dynamics of parties' political positions.

The article is organized as follows: the next section formulates the assumptions of the dynamic state-space model that this article proposes and discusses their motivation. From these assumptions, a method for the estimation of policy positions is derived in a further section. This method combines the estimation of model parameters using maximum marginal likelihood with the prediction of actors' positions using an empirical Bayes method, and employs recent techniques for the approximation of analytically intractable multidimensional integrals, which combine independent sample Monte Carlo methods with automatic adjustment of Monte Carlo sample sizes. These techniques, which are novel in political methodology, have the advantage over the widely used Markov Chain Monte Carlo (MCMC) methods that the assessment of convergence and the determination of the appropriate sample side are much more straightforward.

The model and method are then applied to data from the CMP (Budge et al. 2001; Klingemann et al. 2006; Volkens et al. 2010). This applied section not only presents example estimates, but also shows how the approach taken in this article can be made fruitful for the test of some substantive hypotheses about the evolution of parties' political standpoints. The article concludes with a discussion of its main findings and potential avenues of further research.

This article is supplemented by (1) software to fit the dynamic state-space model and to obtain predictions about the positions of political actors, and by (2) data that result from the application of the methods proposed in this article to the reconstruction of positions of parties from Western Europe and the English-speaking world in the spaces of economic policies and social policies based on CMP data. Both the software and the reconstructed party positions are available for download at the Political Analysis dataverse (Elff 2012) and the author's Web site. In addition, a Supplementary Appendix is available on the Political Analysis Web site and the author's Web site, which gives further details about the methods proposed and employed in the article.

\section{The Construction of the Dynamic State-space Model}

The basic idea behind the model proposed here is similar to the one that motivates the Nominate approach (Poole and Rosenthal 1985) at reconstructing legislators' positions from roll call votes:

\footnotetext{
${ }^{1}$ An extensive discussion of the need for such a measurement model and of what can be learned from the advances in the estimation of ideal from voting can be found in the Supplementary Appendix.
} 
while the Nominate approach rests on the assumption that legislators are more likely to vote in favor of or against a legislative proposal depending on the proximity of the legislative proposal and the status quo toward their own position in a political space, the model proposed here rests on the assumption that actors - parties or candidates - are more likely to talk about or mention favorably in their political texts - e.g., electoral platforms - those policies that are close to their own position.

The positions of the actors expressed in published political texts need not necessarily reflect their own preferences, but they do that only to the degree that the actors are sincere. Yet, even if actors are not sincere and use the expression of position merely to attract voters, a position expressed in a political text can at least be interpreted as the ideal point of the targeted group of voters. That is, even if an electoral platform is only an instrument to attract voters, as in the original Downsian interpretation, a party or candidate will be more likely to favorably mention certain policies the closer they are to the ideal point of the targeted group of voters, and will be less likely to mention them the more distant they are from that ideal point.

Policies can be conceived as belonging to particular policy fields, such as the fields of economic policies, of social policies, of foreign policies, etc. There may be some linkages between parties' or candidates' positions with respect to different policy fields created by their ideology, but this is an aspect relevant for the explanation of position-taking and should be held separate from the aspect of measuring these positions. It is therefore reasonable to assume that each policy has a location in just one of potentially several policy fields touched upon in a political text. Further, policies with regard to, e.g., free market versus state intervention or tax-and-spend versus austerity may happen to be ordered neatly on a single dimension, but there are no reasons a priori to expect that this will always be the case. At the measurement stage, the dimensionality of a policy space should rather be treated as an empirical matter in order to be able to check assumptions with regard to particular policy fields. These considerations motivate the following assumptions:

Assumption 1. Every policy field $f(f=1, \ldots, F)$ - such as economic policy, social policy, foreign policy, etc. - is represented by a $D_{f}$-dimensional Euclidean space (where $D_{f} \geq 1$ ), which henceforth is called a policy space.

Assumption 2. Each policy space $f(f=1, \ldots, F)$ is characterized by several policies $i$ $\left(i=1, \ldots, I_{f}\right)$, which have a specific location in it. The location of policy $i$ is represented by a $D$-dimensional coordinate vector $\alpha_{i}^{(f)}$ with elements $\alpha_{i 1}^{(f)}, \ldots, \alpha_{i D_{f}}^{(f)}$

Assumption 3. The position that a political actor $j$ takes in a policy space $f$ at time $t\left(t=0, \ldots, T_{j}\right)$ by creating or publishing a political text, e.g., an electoral platform, is represented by a $D$-dimensional coordinate vector $\boldsymbol{b}_{j t}^{(f)}$ with elements $b_{j t 1}^{(f)}, \ldots, b_{j t D_{f}}^{(f)}$.

As argued by Laver and Garry (2000), Laver (2001), and Benoit and Laver (2006), the share of a political text devoted to a particular policy may not only reflect - as a "selective emphasis" (Budge and Farlie 1983) - the position that an actor takes in the respective policy field, but also its salience. Among other things, this salience may reflect the urgency of public problems pertinent to this policy field. For example, if economic problems are pressing during an electoral campaign, parties or candidates may devote more space in their electoral platforms to topics of economic policy, whatever their positions are within the economic policy field. That is, the number of words or sentences referring to a particular policy in a political text reflects both the position of an actor relative to this policy and the salience of the policy field to which this policy belongs. Since counts of words or sentences are always nonnegative, the conjunction of salience and of position-related selective emphasis is best expressed as a product. This is formalized by the following assumption:

Assumption 4. Let $Y_{i j t}^{(f)}$ denote the frequency count of tokens (words, sentences, or quasi-sentences) referring to policy $i$, which belongs to policy field $f$, in the text created by actor $j$ at time $t$. The expected value $\mu_{i j t}^{(f)}=\mathrm{E}\left(Y_{i j t}^{(f)}\right)$ of the frequency count can be expressed as the product

$$
\mu_{i j t}^{(f)}=\exp \left(\lambda_{f j t}\right) \exp \left(\eta\left(\left\|\boldsymbol{\alpha}_{i}^{(f)}-\boldsymbol{b}_{j t}^{(f)}\right\|\right)\right),
$$


where $\lambda_{f i t}$ reflects the salience of the policy field $f$ in the political text of actor $j$ at time $t$, and $\eta\left(\left\|\boldsymbol{\alpha}_{i}^{(f)}-\boldsymbol{b}_{j t}^{(f)}\right\|\right)$ is a decreasing function of the distance $\left\|\boldsymbol{\alpha}_{i}^{(f)}-\boldsymbol{b}_{j t}^{(f)}\right\|$ between the location $\boldsymbol{\alpha}_{i}^{(f)}$ of the policy $i$ and the position $\boldsymbol{b}_{j t}^{(f)}$ of the actor $j$ at $t$.

The exponential function present in both factors in equation (1) assures that the expected value $\mu_{i j t}^{(f)}$ will always be nonnegative.

The assumptions so far introduced are fairly general and provide a nonlinear link between the positions of political actors and the frequency in which coding categories occur in a political text, if they can be interpreted as referring to policies. However, the assumptions do not yet allow us, due to their generality, to devise a particular method of reconstructing political positions from political texts. Therefore, another set of more technical assumptions is required, which are discussed in the following. In order to avoid a convoluted notation, the index $f$ for the policy space will be dropped, because almost always only a single policy space is under consideration.

The first of the more technical assumptions concerns the function $\eta()$ in equation (1):

Assumption 5. The function $\eta()$ in equation (1) is a simple quadratic form of its vector argument, given by

$$
\eta(x)=-\frac{1}{2} x^{2}
$$

This functional form is chosen for its simplicity: it is one of the simplest functions, such that $\exp \left(\eta\left(|| \boldsymbol{\alpha}_{i}-\boldsymbol{b}_{j t}||\right)\right)$ is a symmetric twice continuous differentiable function of $\boldsymbol{\alpha}_{i}$ and $\boldsymbol{b}_{j t}$ that decreases with the distance $\left\|\boldsymbol{\alpha}_{i}-\boldsymbol{b}_{j t}\right\|^{2}{ }^{2}$

Models of the Nominate lineage are already probabilistic and thus allow for errors in the vote decisions. In that context, the observations are binary and the appropriate probability model is that of a Bernoulli distribution. In a similar vein, the model proposed here has a probabilistic component to allow for actors being fallible in the production of their text and also allow for measurement error (Benoit, Mikhaylov, and Laver 2009). Since the observations are frequency counts, an appropriate probability model is based on the Poisson family (Slapin and Proksch 2008; Benoit, Mikhaylov, and Laver 2009):

Assumption 6. The frequency count of tokens (words, sentences, or quasi-sentences) $M_{i j t}$ referring to policy $i$ in the text created by actor $j$ at time $t$ has, conditional on the salience of the policy field and the position of the actor, a Poisson distribution with expectation parameter $\mu_{i j t}$ given by equations (1) and (2).

It is well known that the sum of several Poisson-distributed random variables is itself Poisson distributed and that the distribution of these random variables conditional on their sum has a multinomial distribution. This relation between these distributions has been used to model contingency tables via Poisson models (Agresti 2002, 8f); while in the present context, the choice of a Poisson distribution allows to reconstruct positions within a policy field separately from its salience: conditional on their sum, the frequencies of references to policies belonging to a particular policy field follow a multinomial distribution with cell probabilities

$$
\pi_{i j t}=\frac{\mu_{i j t}}{\sum_{h} \mu_{h j t}}=\frac{\exp \left(\lambda_{j t}\right) \exp \left(-1 / 2|| \boldsymbol{\alpha}_{i}-\boldsymbol{b}_{j t} \|^{2}\right)}{\sum_{h} \exp \left(\lambda_{j t}\right) \exp \left(-1 / 2|| \boldsymbol{\alpha}_{h}-\boldsymbol{b}_{j t} \|^{2}\right)}=\frac{\exp \left(-1 / 2|| \boldsymbol{\alpha}_{i}-\boldsymbol{b}_{j t} \|^{2}\right)}{\sum_{h} \exp \left(-1 / 2|| \boldsymbol{\alpha}_{h}-\boldsymbol{b}_{j t} \|^{2}\right)} .
$$

That is, if one conditions on the total number of frequency counts referring to policy counts that belong to a policy field, one can use a multinomial likelihood to estimate the locations of the policies and the positions of the actors separate from the salience of the policy field.

Figure 1 illustrates how the expectation of frequency counts of words or sentences referring to three policy categories is related to the position taken by a political actor in a one-dimensional policy space, where the policy space contains three policy objectives, and the total number of

\footnotetext{
${ }^{2}$ The same functional form was used in the variation on the Nominate model created by Clinton, Jackman, and Rivers (2004) and Martin and Quinn (2002). In the context of ideal point estimation, when only two alternatives are relevant, this functional form allows for further simplifications, in which the right-hand side of the model equation is linear in the relevant parameters (Martin and Quinn 2002; Clinton, Jackman, and Rivers 2004). With more than two alternatives, these simplifications do not work, however.
} 


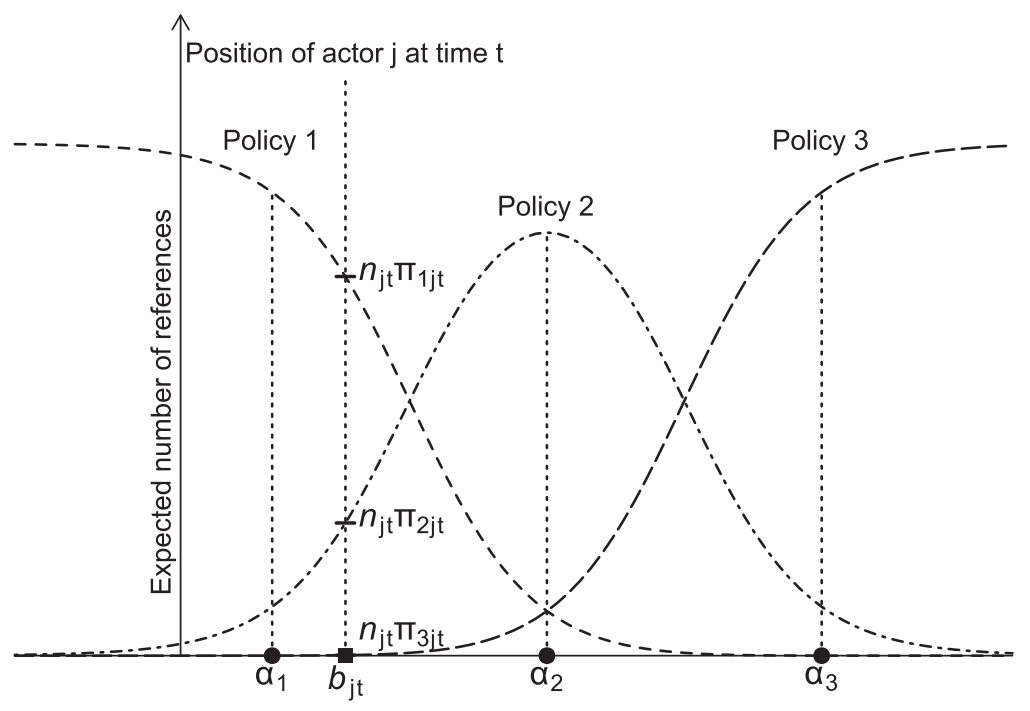

Fig. 1 The relation between the positions of a political actor, the location of three policies, and their emphasis in the actor's political text, if the salience of the corresponding policy field is held constant. The total of the frequencies of the references to the policies in the policy field in question is denoted here by $n_{j t}$, and the cell probabilities as given by equation (3) are denoted by $\pi_{1 j t}, \pi_{2 j t}$, and $\pi_{3 j t}$.

sentences referring to the policy field is held constant (denoted here by $n_{j t}$ ). The horizontal axis represents this one-dimensional policy space, where the three policy objectives with locations $\boldsymbol{\alpha}_{1}, \boldsymbol{\alpha}_{2}$, and $\alpha_{3}$ are represented by round dots on the horizontal axis.

The Assumptions 1 through 6 specify a link between political actors' positions and the frequency in which they refer to policies or policy categories in each of their political texts. They thus constitute a measurement model for the reconstruction of political positions from individual political texts. The absence of restrictions on the location parameters $\alpha_{i}$ (other than some technical assumptions necessary to assure its identification) allows us to test, as an empirical hypothesis, the tacit assumption, underlying the construction of the CMP RiLe index (Budge et al. 2001; Klingemann et al. 2006; Volkens et al. 2010) or of Lowe et al.'s (2011) LogPlanEco index, that policy categories can be collapsed into opposite categories without loss of information.

In addition to its measurement component, the model proposed in this article also contains a dynamic component, which serves two purposes, a substantial and more technical one. The substantial purpose rests on the idea that, unless parties or candidates run for an election for the first time, they do not create their position de novo, but take their position at the previous election as the point of departure, perhaps updating it in reaction to current events, in the light of experience in office or in opposition, or to react to their electoral fortunes in a preceding election (Kollman, Miller, and Page 1992a, 1992b). Especially if parties can be expected to be responsible or if they try to retain a reputation about policy positions (Downs 1957; Alesina 1988; Hinich and Munger 1994), their positions will not change too much over time. The second technical purpose is to take into account a potential autocorrelation between succeeding positions of the same political actor and to achieve a gain in the efficiency and precision in the reconstruction of political positions from exploiting the information contained in the fact that a sequence of political texts originates from a single political actor.

The first two assumptions used to specify the dynamic component are purely technical in nature: Assumption 7. The positions of $\boldsymbol{b}_{j t}$, the political actors, have a common expectation $E\left(\boldsymbol{b}_{j t}\right)=\boldsymbol{\beta}$.

Assumption 8. The deviation $\boldsymbol{u}_{j 0}=\boldsymbol{b}_{j 0}-\boldsymbol{\beta}$ of an political actor's position $\boldsymbol{b}_{j 0}$ from the mean $\boldsymbol{\beta}$ at time $t=0$, when for the first time a political text is available from the actor, has a normal distribution with zero mean and variance matrix $\boldsymbol{\Sigma}_{0}$, so one can write

$$
\boldsymbol{b}_{j 0}=\boldsymbol{\beta}+\boldsymbol{u}_{j 0}, \quad \boldsymbol{u}_{j 0} \sim \mathcal{N}\left(\mathbf{0}, \boldsymbol{\Sigma}_{0}\right) .
$$


Assumptions 7 and 8 assure that the distribution of a finite sequence of an actor's political positions has a finite mean and a finite variance, as long as positional changes have a finite variance. The common expectation $\boldsymbol{\beta}$ of the actors' positions can be interpreted as the "political center" of the policy space in question.

The next assumption describes the dynamics and is essentially the same as the assumption of Martin and Quinn (2002) about the evolution of legislators' ideal points:

Assumption 9. The positions of the political actors take the form of a VAR(1) vector autoregression. That is, conditional on the position at a previous time point $t-1$, the deviation $\boldsymbol{u}_{t j}=\boldsymbol{b}_{j t}-\boldsymbol{\beta}$ of the position $\boldsymbol{b}_{j t}$ of a political actor $j$ at time $t$ from the mean of the positions has a normal distribution with mean $\boldsymbol{\Gamma} \boldsymbol{u}_{j t-1}$ and variance matrix $\Sigma_{1}$, where $\boldsymbol{u}_{j t-1}=\boldsymbol{b}_{j t-1}-\boldsymbol{\beta}$ and $\Gamma$ is a matrix of autoregression slopes. One can therefore write

$$
\boldsymbol{b}_{j t}=\boldsymbol{\beta}+\boldsymbol{u}_{t j}, \quad \boldsymbol{u}_{t j}=\boldsymbol{\Gamma} \boldsymbol{u}_{j t-1}+\boldsymbol{e}_{t j}, \quad \boldsymbol{e}_{t j} \sim \mathcal{N}\left(\mathbf{0}, \boldsymbol{\Sigma}_{1}\right) .
$$

The technical implications of Assumptions 7-9 will become clear in the following section on estimation. In the present context, however, it should be noted that these assumptions allow us to formalize various substantive hypotheses about the dynamics of political actors' positions. For example, if all elements of the autoregression matrix $\boldsymbol{\Gamma}$ are zero, the positions of any actor will be just memory-less fluctuations around an overall mean $\boldsymbol{\beta}$. If $\boldsymbol{\Gamma}$ is an identity matrix, then the positions will be characterized as a nonstationary random walk, and if $\Gamma$ is diagonal with elements smaller than unity, the actors' positions will form a time series that is stationary relative to the actors' initial positions $\boldsymbol{b}_{j 0}=\boldsymbol{\beta}+\boldsymbol{u}_{j 0}$. In particular, this will mean that actors will on average tend to take less extreme stances when they update their positions relative to a prior election.

\section{Estimating Model Parameters and Predicting Political Positions}

The latent state-space model of actors' political positions specified in the previous section treats positions as latent data and not as parameters. This has the crucial advantage that it avoids an incidental parameters problem. If the positions were parameters, then any new political text from which an actor's positions could be derived would create a new set of parameters. Treating the positions as latent data, however, means that they are reconstructed after the model parameters are estimated. This reconstruction takes the form of moments of the predictive distribution of the positions based on the estimated model.

Estimates of the model parameters are obtained by maximizing the marginal likelihood

$$
\mathcal{L}(\boldsymbol{y} ; \psi)=\prod_{j} \int f\left(\boldsymbol{y}_{j} \mid \boldsymbol{b}_{j} ; \psi\right) f\left(\boldsymbol{b}_{j} ; \psi\right) \mathrm{d} \boldsymbol{b}_{j},
$$

which by construction does not depend on unobserved data, but only on the parameters and the observed frequency counts $\boldsymbol{y}_{j}$ of coding categories. ( $\boldsymbol{y}_{j}$ is formed by "stacking" the vectors $\boldsymbol{y}_{j 0}, \ldots, \boldsymbol{y}_{j T_{j}}$, where $\boldsymbol{y}_{j t}$ is the vector of observed frequency counts of the coding categories in the political text produced by actor $j$ at time $t$ and $\boldsymbol{b}_{j}$ is analogously formed from the positions $\boldsymbol{b}_{j t}$. ) The vector $\boldsymbol{\psi}$ in equation (6) contains the parameters of the model, namely: (1) the coordinate vectors $\alpha_{1}, \ldots, \alpha_{I}$ of the locations of the $I$ policies in a given policy space, (2) a grand mean $\boldsymbol{\beta}$ of actors' positions in the policy space, (3) the autoregression slope matrix $\boldsymbol{\Gamma}$, and (4) the variance matrices $\boldsymbol{\Sigma}_{0}$ of the initial positions and $\boldsymbol{\Sigma}_{1}$ of the position updates. Likelihoods of the form (6) are typically maximized using an expectation-maximization (EM) algorithm because a straightforward Newton-Raphson algorithm can be formally unwieldy and numerically unstable (Dempster, Laird, and Rubin 1977; Little and Rubin 2002). Details of the EM algorithm for the present case are discussed in the Supplementary Appendix of this article.

Once maximum likelihood estimates $\widehat{\psi}$ are obtained for the model parameters, positions can be reconstructed from the expectation of the predictive distribution, whereas measures 
of uncertainty about the positions can be derived from the variance or the quantiles of the predictive distribution. This predictive distribution of the positions takes the form

$$
f\left(\boldsymbol{b}_{j} \mid \boldsymbol{y}_{j} ; \widehat{\psi}\right)=\frac{f\left(\boldsymbol{y}_{j} \mid \boldsymbol{b}_{j} ; \widehat{\psi}\right) f\left(\boldsymbol{b}_{j} ; \widehat{\psi}\right)}{\int f\left(\boldsymbol{y}_{j} \mid \boldsymbol{b}_{j} ; \widehat{\psi}\right) f\left(\boldsymbol{b}_{j} ; \widehat{\psi}\right) \mathrm{d} \boldsymbol{b}_{j}} .
$$

Quite obviously, this predictive distribution has the form of a posterior distribution in the sense of Bayesian inference. However, since the parameters of the prior distribution are estimated from empirical data, this is an empirical Bayes posterior.

The marginal likelihood, the intermediate steps of the EM algorithm, and the posterior distribution of actors' positions involve integrals that do not have a closed-form solution. Therefore, these integrals can be computed only using methods of numeric approximation. Since standard quadrature methods are infeasible for the highly multidimensional integrals, a Monte Carlo method of approximation is used (Ripley 1987; Gentle 2003; Robert and Casella 2004), which transforms the EM algorithm into a Monte Carlo EM (MCEM) algorithm (Wei and Tanner 1990). An efficient method to approximate the integral in the marginal likelihood and the steps of the MCEM algorithm are based on importance sampling (see, e.g., Robert and Casella 2004, 90ff, 174ff): in a first step, $R$ random vectors $\boldsymbol{b}_{j}^{*(r)}(r=1, \ldots, R)$ are generated from a proposal distribution with density $f_{\text {pro }}()$. In the second step, the integral is approximated by the weighted mean:

$$
\int f\left(\boldsymbol{y}_{j} \mid \boldsymbol{b}_{j} ; \boldsymbol{\psi}\right) f\left(\boldsymbol{b}_{j} ; \boldsymbol{\psi}\right) g\left(\boldsymbol{b}_{j}\right) \mathrm{d} \boldsymbol{b}_{j} \approx \frac{1}{R} \sum_{r=1}^{R} \frac{f\left(\boldsymbol{y}_{j} \mid \boldsymbol{b}_{j}^{*(r)} ; \boldsymbol{\psi}\right) f\left(\boldsymbol{b}_{j}^{*(r)} ; \boldsymbol{\psi}\right)}{f_{\mathrm{pro}}\left(\boldsymbol{b}_{j}^{*(r)}\right)} g\left(\boldsymbol{b}_{j}^{*(r)}\right),
$$

where the weights $f\left(\boldsymbol{y}_{j} \mid \boldsymbol{b}_{j}^{*(r)} ; \boldsymbol{\psi}\right) f\left(\boldsymbol{b}_{j}^{*(r)} ; \psi\right) / f_{\text {pro }}\left(\boldsymbol{b}_{j}^{*(r)}\right)$ are the so-called importance weights. Following Booth and Hobert (1999) and Caffo, Jank, and Jones (2005), the proposal distribution used is a (shifted and rescaled) multivariate Student's distribution, which is derived from the second-order Taylor expansion of the integrand. ${ }^{3}$

While quite efficient, importance sampling is less useful for obtaining quantiles of the posterior distribution of actors' positions, because a Monte Carlo approximation of quantiles requires unweighted samples. Therefore, the posterior distribution of the actors' positions is approximated using samples based on an accept-reject method (Robert and Casella 2004, 41). The difference between importance sampling and the accept-reject method is that random vectors $\boldsymbol{b}_{j}^{*(r)}$ generated from the proposal distribution are not weighted, but resampled with acceptance probability proportional to $f\left(\boldsymbol{y}_{j} \mid \boldsymbol{b}_{j}^{*(r)} ; \widehat{\psi}\right) f\left(\boldsymbol{b}_{j}^{*(r)} ; \widehat{\psi}\right) / f_{\text {pro }}\left(\boldsymbol{b}_{j}^{*(r)}\right)$. The accept-reject method is computationally more demanding than the importance-sampling method, which is why the latter is used in the iterations of the MCEM algorithm.

It should be noted that the Monte Carlo methods just mentioned, unlike MCMC methods (e.g., Martin and Quinn 2002; Clinton, Jackman, and Rivers 2004), are based on mutually independent samples. A particular advantage of independent sampling is that Monte Carlo error can be assessed directly and the Monte Carlo sample size can be adapted to the precision required by the respective stages of the MCEM algorithm. Booth and Hobert (1999) and Caffo, Jank, and Jones (2005) derive methods for the automatic adjustment of the Monte Carlo sample sizes during the progression of MCEM iterations, to balance computational cost with precision. Thus, an MCEM algorithm can start with relatively small samples, the sizes of which will increase as the MCEM progresses further and requires a higher precision from the Monte Carlo approximation. The implementation of the MCEM algorithm for this article uses Caffo, Jank, and Jones's (2005) method of sample size adjustment. Details can be found in the Supplementary Appendix of this article.

The methods just described are implemented in $R$ ( $\mathrm{R}$ Development Core Team 2011) with some extensions in $\mathrm{C}++$ with the help of the Rcpp (Eddelbuettel and François 2011) and RcppArmadillo (Francois, Eddelbuettel, and Bates 2011) packages. The software implementing these methods is available for download on the Political Analysis dataverse (Elff 2012) and on the author's Web site.

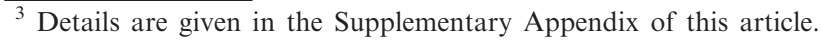


Table 1 The dynamic state-space model of parties' positions in the economic policy space

\begin{tabular}{lr}
\hline Locations of policy categories & Estimate $(S E)$ \\
\hline Nationalization & $-1.853(0.008)$ \\
Controlled economy & $-1.414(0.007)$ \\
Econ. planning & $-1.166(0.007)$ \\
Market regulation & $0.057(0.008)$ \\
Incentives & $0.885(0.008)$ \\
Econ. orthodoxy & $1.642(0.007)$ \\
Free enterprise & $1.849(0.007)$ \\
\hline Positions taken in platforms & $0.767(0.034)$ \\
\hline Mean & $0.806(0.013)$ \\
Autoregression slope & $1.032(0.069)$ \\
Co-)Variances & $0.302(0.014)$ \\
$\quad$ Between actors & \\
Between time points & $-65,363.90$ \\
\hline Summary statistics & $102,466.00$ \\
\hline Log-likelihood & 3334 \\
Deviance & 153,411 \\
\hline Totals & 840 \\
\hline Number of parties & \\
\hline Number of platforms & \\
\hline
\end{tabular}

Note. The table shows maximum likelihood estimates and standard errors in parentheses, as well as summary statistics and totals.

\section{Reconstructing Positions of Political Parties Based on Data from the Manifesto Project}

In the following, the model and the estimation methods described in the previous two section are applied to the reconstruction of positions taken by parties from contemporary democracies in the electoral platforms covered by the CMP (Budge et al. 2001; Klingemann et al. 2006; Volkens et al. 2010). The CMP has been collecting, coding, and analyzing electoral platforms of political parties from most of the Western democracies, a collection that ranges from the most recent past back to the early post-World War II era, and for the United States even further back. As a result of their efforts, the CMP has contributed a data set that for each electoral platform contains, along with other information, the percentages of quasi-sentences that refer to each of the fiftysix broad policy categories, which the CMP refers to as "policy objectives." ${ }^{4}$ In order to apply the estimation procedure described in the previous section, these percentages were transformed back into frequency counts, making use of the information in the CMP data set about the total number of quasi-sentences in each coded electoral platform. The categories used for the reconstruction of the space of economic policies are "Nationalization" (the corresponding variable in the CMP data set is Per413), "Controlled economy" (Per412), "Economic planning" (Per404), "Market regulation" (Per403), "Incentives" (Per402), "Economic orthodoxy" (Per414), and "Free enterprise" (Per405).

Table 1 gives the estimates of the model parameters of the dynamic state-space model for the space of economic policies. The policy space is considered as one-dimensional, contrasting typical "laissez-faire" positions to "state-interventionist" positions on an economic left-right dimension.

\footnotetext{
${ }^{4}$ The author of this article is affiliated neither with the Manifesto Research Group nor with the Comparative Manifestos Project and gratefully acknowledges their contribution in making these data available to the public. This means, of course, that the investigators of the Comparative Manifestos Project are not responsible for any errors in the analysis and interpretation of these data in this article. Data and $R$ scripts to replicate the analyses conducted in this section are available on the Political Analysis dataverse: http://hdl.handle.net/1902.1/19198 (Elff 2012).
} 
In order to assure the identification of the model, the coordinate values of the policy objectives along the economic left-right dimension are constrained to sum to zero. Since the expected value of the distribution of the political positions has an estimate different from zero, one should compare the locations of the policy categories with the mean of the distribution of the political positions exhibited in the political texts rather than in terms of the sign.

A comparison of the locations of the policy objectives with the mean of the distribution of the positions is consistent with the notion that the objectives can be distinguished into "leftist" ones and "rightist" ones. The interventionist objectives "Nationalization," "Controlled economy," "Economic planning," and "Market regulation" are located at the left of the mean of the distribution of the political positions, whereas the more laissez faire-oriented objectives "Incentives," "Economic orthodoxy," and "Free enterprise" are located to the right of the mean of the political positions. On the other hand, in so far as the estimated locations of the policy categories vary in their "extremity," they undermine the notion that there is a clear-cut dichotomy between "leftist" and "rightist" policy objectives - a notion that forms the bases of many of the indices of ideological or policy positions (e.g., Laver and Garry 2000; Budge et al. 2001; Klingemann et al. 2006; Lowe et al. 2011). In fact, a Wald test of the hypothesis that the three "leftist" objectives have essentially the same location leads to its rejection, as does a test of the hypothesis that the three "rightist" objectives have the same location (details of the test are reported in the Supplementary Appendix of this article).

The estimates presented in Table 1 also have implications for the dynamics of change in the positions of parties in the space of economic policy. The autoregression parameter is statistically different from zero and positive, which indicates that parties take their previous positions as points of departure when they choose a position on occasion of an election instead of choosing the position each time de novo. This supports the notion that parties avoid to completely abrogate past positions in order to retain their credibility (Downs 1957; Alesina 1988; Hinich and Munger 1994). Further, the autoregression parameter is smaller than unity, which means that parties' updated positions are slightly closer to the center of the distribution of parties' position. This supports the notion that party competition is centripetal, even though parties change their positions only adaptively (Kollman, Miller, and Page 1992a, 1992b).

The previous discussion shows that the model proposed in this article can be used as a measurement model to check the implicit notions behind some left-right indices used in the literature and to make general statements about the general pattern of party competition. But it can also be used to reconstruct the positions of political actors at specific points in time. As was explained in the previous section, this reconstruction is based on the posterior distribution (in an empirical Bayes sense) of the positions given the data contained in the coded political texts. This is illustrated with the example of positions of the Labour Party of the United Kingdom on occasion of the parliamentary elections of 1964 and 1997 in Fig. 2. The means of the posterior distribution of its positions in 1964 and 1997 are indicated by the round dots on the horizontal axis. The shape of the posterior density of the position in 1964 and of the position in 1997 are each approximated by a kernel density estimate based on 5000 sample values from the posterior distribution, which is indicated by the solid curves in the diagram. The dark gray areas each represent approximately $95 \%$ of the posterior probability mass of one of the positions and are enclosed by the $2.5 \%$ and $97.5 \%$ sample quantiles of the simulated values. Each pair of quantiles thus encloses a $95 \%$ posterior credibility or prediction interval. To ease the interpretation, the diagram also shows the location of the seven policy objectives that belong to this political space.

Figure 2 makes clear that the information the electoral platforms provide about the positions of the Labour Party of these two years is not perfect, and that the uncertainty about Labour's position in 1964 is higher than the uncertainty about their position in 1997. Nevertheless, the figure also underlines the distinctiveness of the two positions: the kernel density estimates hardly overlap. This nicely reflects the change that Labour's position underwent between 1964, when the level of class voting in Britain reached a peak (Heath et al. 1991; Weakliem and Heath 1999), and 1997, after the party's rebranding into "New Labour" (Evans, Heath, and Payne 1999; Fielding 2003).

A wider net is cast by Fig. 3, which shows the development of the economic policy positions of major conservative and social democratic parties from six Western European countries, namely 


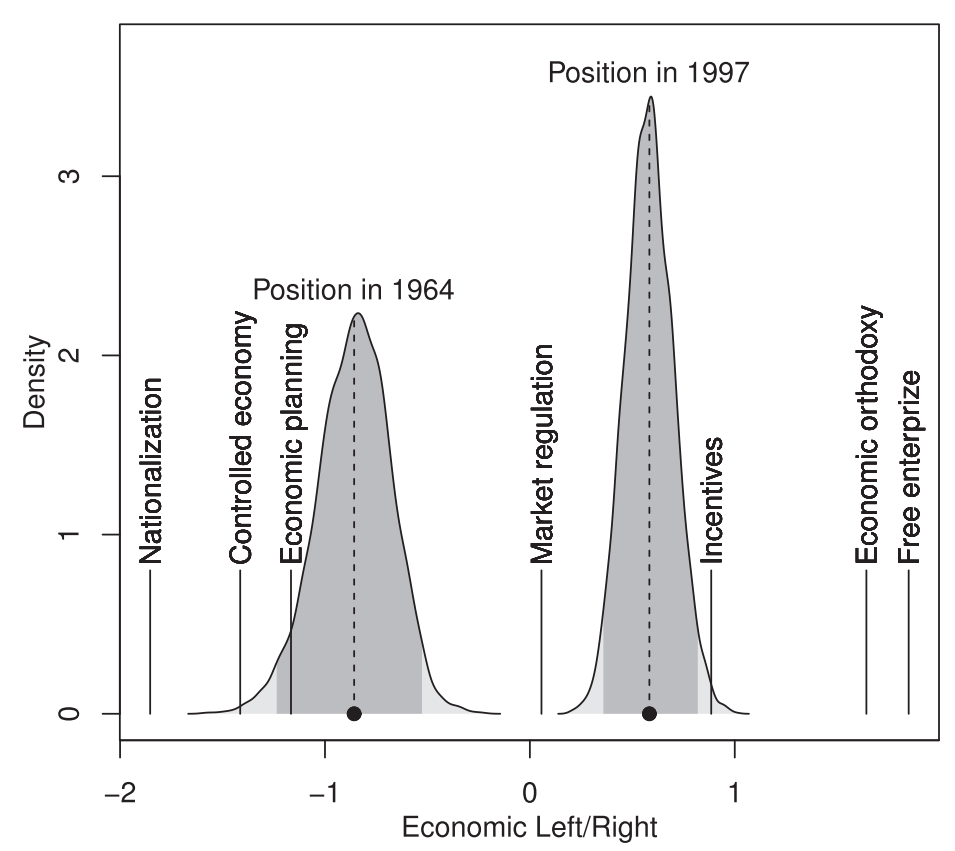

Fig. 2 The posterior distribution of the position of the Labour Party in 1964 and 1997 and the location of economic policy categories in the economic policy space. The curves represent kernel density estimates of the simulated posterior distribution for both time points, where the simulation sample size is 5000 . The dark gray regions correspond to approximately $95 \%$ probability mass of the posterior distribution and are limited by the simulated $2.5 \%$ and $97.5 \%$ quantiles of the posterior distribution. The black dots represent the mean of the posterior distribution for both time points.

France, Germany, the Netherlands, Sweden, Spain, and the United Kingdom. The lines shown in the panels of this figure connect the posterior means of the positions of each party at each election in the respective countries. The gray areas are prediction bands constructed from the $95 \%$ credibility intervals of the positions. As can be observed in Fig. 3, the positions of conservative parties and of the social democratic parties converge towards each other in each of the six countries, a pattern that is consistent with an estimate of autoregression parameter that is smaller than unity in Table 1. The parties in each country obviously get more similar in terms of their positions on economic policy, consistent with arguments brought forward by Elff (2009) that whatever decline in class voting that has occurred in Western European countries after 1975 can mostly be attributed to a decline in political divisions between the "workers' parties" of the traditional left and the "bourgeois parties" of the political center-right. ${ }^{5}$

If one compares the changes in the positions of the parties in Fig. 3 with their confidence bands, one may wonder if these changes are genuine policy shifts and if it is possible to test whether they are statistically significant. However, this question cannot be answered using classical methods of hypothesis testing, since the parties' positions are not parameters but unobserved data. Yet, it is possible to devise a criterion for the statistical significance of a position change based on its posterior distribution: if the probability that the difference between the position at $t$ and the position at $t-1$ is larger than zero or smaller than zero with probability of at least $95 \%$, one can declare this difference as significant. This criterion is satisfied if zero is either smaller than the $5 \%$ quantile of the posterior distribution of the difference or larger than the $95 \%$ quantile. Once a Monte Carlo approximation of the posterior distribution of the individual positions is available, the

\footnotetext{
${ }^{5}$ It should be noted that the apparent peculiar movement in the 2000s of the Swedish parties toward the left also shows up if one uses, e.g., Lowe et al.'s (2011) "LogPlanEco" index. For details, see the Supplementary Appendix of this article.
} 


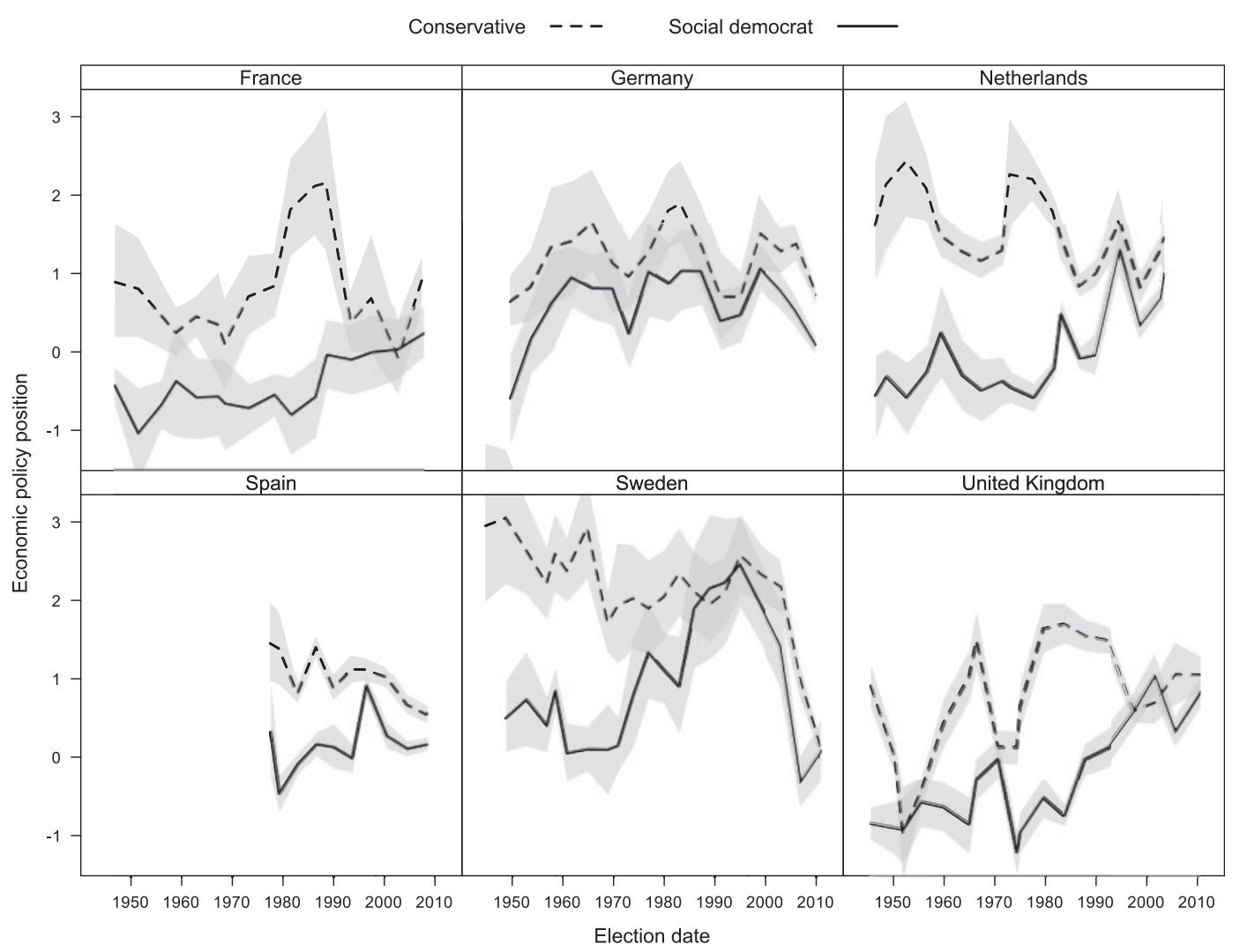

Fig. 3 A comparison of the evolution of the political positions of major conservative and social democratic parties of Western Europe. The conservative parties considered here are the Gaullistes of France (in their various guises as Rassemblement pour la République (RPR), Union pour un Mouvement Populaire (UMP), etc.), the Christlich Demokratische Union (CDU) and Christlich-Soziale Union (CSU) of Germany, the Volkspartij voor Vrijheid en Democratie (VVD) of the Netherlands, the Alianza Popular/Partido Popular of Spain, the Moderata samlingspartiet of Sweden, and the Conservatives of the United Kingdom. The social democratic parties considered are the Parti Socialiste of France, the Sozialdemokratische Partei Deutschlands (SPD) of Germany, the Partij van de Arbeid (PvdA) of the Netherlands, the PSOE of Spain, the Sveriges Socialdemokratiska arbetarparti of Sweden, the Labour Party of the United Kingdom. The dashed and solid lines connect the simulated posterior expectations of the parties' positions on occasion of the respective elections. The gray areas are $95 \%$ prediction bands based on the simulated $2.5 \%$ and $97.5 \%$ quantiles of the posterior distribution of the parties' positions.

approximation of the posterior distribution of the difference of successive positions is straightforward. If $\boldsymbol{b}_{j t}^{*(r)}$ is the $r$ th sample value from the posterior of actor $j$ 's position at time $t$, then $\boldsymbol{b}_{j t}^{*(r)}-\boldsymbol{b}_{j, t-1}^{*(r)}$ is the $r$ th sample value from the posterior distribution of the difference of the positions between $t$ and $t-1$. The quantiles of the posterior distribution of the difference thus can be approximated using the sample quantiles of these simulated differences.

Figure 4 illustrates this procedure with the example of the French Socialist Party and the Labour Party of the UK. These parties are chosen because in Fig. 3, the positions of the French parties exhibit relatively wide prediction intervals, whereas the positions of the British parties show pronounced changes in their positions, yet prediction intervals of relative moderate width. The dots in Fig. 4 represent the posterior expectation of a policy shift, that is, of the difference of the party's position at the election in question relative to the position at the previous election. The gray bars connect the $5 \%$ and $95 \%$ quantiles of Monte Carlo samples of size 5000 from the posterior distributions. In this figure, a policy shift is significant, if the gray bars do not cross the zero line.

As becomes obvious from Fig. 4, most position changes by the French Socialist Party are not statistically significant, except for two elections, the election of 1951, where it moved to the left, and the election of 1988, where it moved to the right. In comparison, the positions of the British Labour 


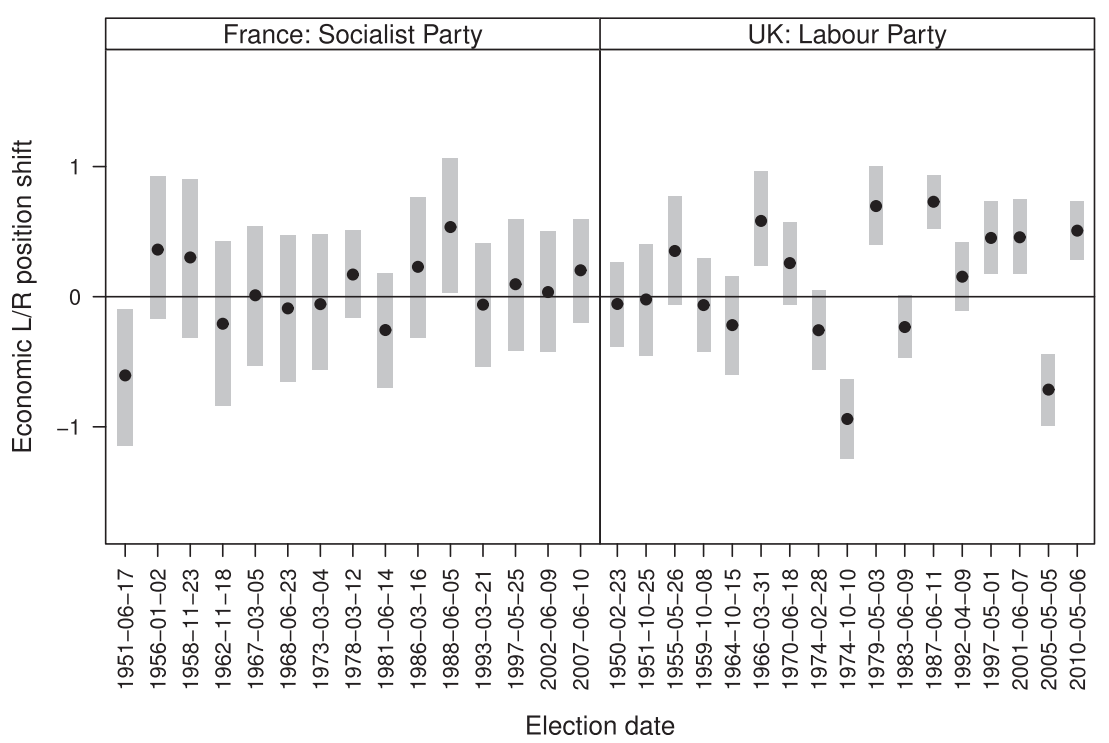

Fig. 4 Reconstructed policy shifts of the French Socialist Party and the Labour Party of the UK. Dots represent the posterior expectations of policy shifts. Gray bars connect 5\% with 95\% quantiles of Monte Carlo samples from the posterior distribution of the policy shifts. The sizes of the Monte Carlo samples are 5000 .

Party appear much more volatile, especially after 1966, with policy shifts on the occasion of eight different elections. Interestingly, the shift of Labour in 1997 does not appear particularly pronounced. Instead, the Labour Party shows stronger movements toward the right in 1987, and in 2005, it makes an excursion to the left. Apparently, Tony Blair's leadership had less consequence in a single shift than in a more or less gradual, though interrupted, drift to the right.

The model of parties' positions in the space of economic policy discussed so far rests on a unidimensional conception. Yet, it is also possible to conceive of political spaces that contain more than one dimension. For example, Elff (2009) considers a space of noneconomic domestic policies that is two-dimensional, where the first dimension contrasts liberal to authoritarian positions and the second dimension contrasts positions favoring moral permissiveness to positions emphasizing traditional morals or ways of life. The policy objectives that belong to the political space so conceived are "Democracy" (Per202), "Freedom and human rights" (Per201), "Law and order" (Per605), and "National way of life (positive)" (Per601) (i.e., positive references to the typical way of life of the majority nationality or ethnicity in a country), "Traditional morality (positive)" (Per603), and "Traditional morality (negative)" (Per604) (i.e., references to policies that favor secular or nontraditional lifestyles, such as divorce and abortion). Table 2 shows the parameter estimates of the locations of these policy objectives in the political space under consideration, as well as estimates of parameters that describe the distribution of parties' position in this political space. In order to make the model identified and to allow for an interpretation of the coordinates of the policy objectives and positions in terms of an authoritarian-liberal and a traditionalistpermissive dimension, some of the coordinate values of the objectives are fixed at zero. These fixed values are not presented in the table.

The estimates of the location parameter suggest that there is a clear distinction between the "authoritarian" objectives "National way of life (positive)" and "Law and order" on the one hand, and "Democracy" and "Freedom and human rights" on the other hand; and that there is also a clear distinction between the objectives "Traditional morality (positive)" and "Traditional morality

\footnotetext{
${ }^{6}$ The Manifesto data also record negative references to the "national way of life"; yet, these are so rarely used in the electoral platforms that they are not considered for the reconstruction of the policy space of noneconomic domestic policy.
} 
Table 2 A dynamic state-space model of parties' positions in the space of noneconomic domestic policies

\begin{tabular}{|c|c|c|c|c|}
\hline \multirow[b]{2}{*}{ Locations of policy categories } & \multicolumn{2}{|c|}{ Authoritarian/liberal } & \multicolumn{2}{|c|}{ Traditionalist/permissive } \\
\hline & Estimate & $(S E)$ & Estimate & $(S E)$ \\
\hline Freedom and human rights & -1.206 & $(0.006)$ & 0.729 & $(0.006)$ \\
\hline Democracy & -1.145 & $(0.007)$ & 0.092 & $(0.007)$ \\
\hline Traditional moral. neg. & & & -2.728 & $(0.009)$ \\
\hline Traditional moral. pos. & & & 1.907 & $(0.006)$ \\
\hline Law and order & 0.766 & $(0.007)$ & & \\
\hline National way pos. & 1.585 & $(0.006)$ & & \\
\hline \multicolumn{5}{|l|}{ Positions taken in platforms } \\
\hline Means & -0.278 & $(0.043)$ & 0.254 & $(0.032)$ \\
\hline \multicolumn{5}{|l|}{ Autoregression slopes } \\
\hline Authoritarian/liberal & 0.790 & $(0.018)$ & 0.046 & $(0.020)$ \\
\hline Traditionalist/modernist & 0.082 & $(0.015)$ & 0.751 & $(0.018)$ \\
\hline \multicolumn{5}{|l|}{$(\mathrm{Co}-)$ Variances } \\
\hline \multicolumn{5}{|l|}{ Between actors } \\
\hline Authoritarian/liberal & 0.963 & $(0.062)$ & 0.318 & $(0.037)$ \\
\hline Traditionalist/modernist & 0.318 & $(0.037)$ & 0.586 & $(0.035)$ \\
\hline \multicolumn{5}{|l|}{ Between time points } \\
\hline Authoritarian/liberal & 0.355 & $(0.017)$ & 0.085 & $(0.011)$ \\
\hline Traditionalist/modernist & 0.085 & $(0.011)$ & 0.267 & $(0.012)$ \\
\hline \multicolumn{5}{|l|}{ Summary statistics } \\
\hline Log-likelihood & $-46,509.1$ & & & \\
\hline Deviance & $66,325.6$ & & & \\
\hline \multicolumn{5}{|l|}{ Totals } \\
\hline Number of parties & 840 & & & \\
\hline Number of platforms & 3334 & & & \\
\hline Total number of quasi-sentences & 170,063 & & & \\
\hline
\end{tabular}

Note. The table shows maximum likelihood estimates and standard errors in parentheses, as well as summary statistics and totals.

(negative)." With respect to the distribution of parties' positions in this political space, one finds, similar to Table 1, a considerable autocorrelation in the positions of the parties along the two axes, and the variance of parties' initial positions is considerably larger than the variance of changes in parties' positions. While there is a clear positive covariance in the parties' initial positions along the two dimensions, parties' movements along the two dimensions are almost independent: the autoregression of positions along the authoritarian-liberal axis on positions along the traditionalist-permissive axis is close to zero, as is the converse autoregression of positions on the traditionalist-permissive axis on positions along the authoritarian-liberal axis. Further, the covariance of the innovations in the positions along the two axes is also close to zero. The autocorrelation of the positions along the two axes is nevertheless sufficiently different from unity that there is a tendency of convergence in the parties' positions in this political space.

The reconstruction of parties' political positions based on coded political texts can of course only be as good as the categories used in coding them. Indeed, the coding categories have been occasionally criticized as not very well capturing what is really going on in parties' electoral platforms (Laver and Garry 2000). Improved coding schemes and procedures may thus enhance the precision and reliability of political positions reconstructed using the method proposed in this article or any other method. Nevertheless, the applications discussed in this section demonstrate how the dynamic state-space model can be used to test theoretically motivated hypotheses and to come to substantially interesting conclusions about the dynamics of parties' political positions. 


\section{Discussion}

The present article proposes a dynamic state-space model of political texts, which combines a measurement component with a dynamic component. The measurement component provides a linkage between positions in a policy space taken by political actors, such as parties or candidates, with observable data in the form of frequency counts of categories in coded political texts, especially of references to policies or broader policy categories in electoral platforms. At the same time, it provides a way to separate the salience of policy areas from actors' positions within these areas. The dynamic component of the model allows us to describe in terms of a finite set of parameters how actors' positions evolve over time. The article further shows how the parameters of the model can be estimated based on the maximum likelihood principle, which enables the use of likelihood-based inference for the empirical assessment of hypotheses about the dynamics of party competition. It also demonstrates how the positions of political actors at particular points in time can be reconstructed from their empirical Bayes posterior distribution and how the uncertainty about this reconstruction can be assessed.

The application of the model to data from the CMP leads to both methodological and substantive conclusions: first, the political objectives as they form the core of the coding scheme of the CMP cannot be simply categorized into homogeneous blocks of "leftist" or "rightist" objectives. Instead, policy objectives vary in the "extremity" of their locations in a policy space. Second, the evolution of parties' positions shows a general tendency of convergence; yet, the positions that parties take in their electoral platforms are not independent from positions taken on earlier occasions. Third, there is evidence that, in terms of noneconomic domestic policy, one should distinguish between an authoritarian-liberal and a traditionalist-permissive dimension.

The dynamic state-space model of coded political texts addresses issues that arise after political texts have been segmented into syntactic or semantic tokens and after these tokens have been grouped into categories. That is, issues such as the appropriate construction of coding categories (Laver and Garry 2000) or the reliability of the segmentation of political texts into tokens and the classification of tokens into categories (Benoit, Mikhaylov, and Laver 2009; Mikhaylov, Laver, and Benoit 2012) remain. This situation is similar to the one in psychological testing. IRT can be used to formalize the relation between indicators and psychological concepts they are supposed to measure, and to check the quality of these indicators using the toolbox of likelihood-based inference (McDonald 1999; for a recent example in political science, see Stegmueller 2011). But the construction or choice of test items is a task that requires substantive psychological knowledge and experience. Similarly, the model proposed in this article can contribute to the assessment of coding procedures of political texts as well as to the use of coded political texts for answering substantive research questions. Yet, the model neither makes considerations about the validity and reliability of coding procedures redundant nor is made redundant by the solution of problems pertaining to such procedures.

The dynamic component of the proposed model can be brought to its full potential only if a sufficiently large number of coded political texts is available. So far, the CMP (Budge, Robertson, and Hearl 1987; Budge et al. 2001; Klingemann et al. 2006; Volkens et al. 2010) is the only source of such data available so far. This is why the model proposed here is applied to the CMP data. But, this does not mean that the CMP data are the only data set to which it can be applied. The model can be easily adapted to more limited data sets. If only a cross-section of coded texts is available, the parameters relevant for the dynamic component of the model cannot be estimated. Yet, if the number of texts in this cross-section is large enough, one can still estimate the variance parameter matrix $\boldsymbol{\Sigma}_{0}$ and obtain the posterior mean prediction of an appropriately simplified posterior distribution. If the number of texts is too small to allow for the estimation of $\boldsymbol{\Sigma}_{0}$, one may prefer to treat the actors' positions $\boldsymbol{b}_{j}$ as parameters and, instead of integrating them out for the construction of the marginal likelihood, estimate them directly by maximum likelihood. If the number of categories is very large, or if tokens are not coded into categories, but are used "as is," one may consider treating the locations of the tokens or categories as latent data and to consider a maximum marginal likelihood approach where these locations are integrated out. That is, the estimation techniques discussed in this article have a wider potential use than only for the dynamic state-space model proposed. 


\section{References}

Adams, J., and S. Merrill III. 2009. Policy-seeking parties in a parliamentary democracy with proportional representation: A valence-uncertainty model. British Journal of Political Science 39(3):539-58.

Agresti, A. 2002. Categorical data analysis. 2nd ed. New York: Wiley.

Alesina, A. 1988. Credibility and policy convergence in a two-party system with rational voters. American Economic Review 78(4):796-805.

Benoit, K., and M. Laver. 2006. Party policy in modern democracies. London/New York: Routledge.

Benoit, K., S. Mikhaylov, and M. Laver. 2009. Treating words as data with error: Uncertainty in text statements of policy positions. American Journal of Political Science 53(2):495-513.

Booth, J. G., and J. P. Hobert. 1999. Maximizing generalized linear mixed model likelihoods with an automated Monte Carlo EM algorithm. Journal of the Royal Statistical Society, Series B (Statistical Methodology) 61(1):265-85.

Budge, I., and D. Farlie. 1983. Party competition: selective emphasis or direct confrontation? An alternative view with data. In Western European party systems continuity and change, eds. H. Daalder and P. Mair, 265-305. Beverly Hills, CA: Sage.

Budge, I., H.-D. Klingemann, A. Volkens, J. Bara, and E. Tanenbaum. 2001. Mapping policy preferences: Estimates for parties, electors, and governments 1945-98. Oxford: Oxford University Press.

Budge, I., D. Robertson, and D. Hearl. 1987. Ideology, strategy, and party change: Spatial analysis of post war election programs in 19 democracies. Cambridge, MA: Cambridge University Press.

Caffo, B. S., W. Jank, and G. L. Jones. 2005. Ascent-based Monte Carlo expectation-maximization. Journal of the Royal Statistical Society, Series B (Methodological) 67(2):235-51.

Clinton, J., S. Jackman, and D. Rivers. 2004. The statistical analysis of roll call data. American Political Science Review 98(2):355-70.

Dempster, A. P., N. M. Laird, and D. B. Rubin. 1977. Maximum likelihood from incomplete data via the EM algorithm. Journal of the Royal Statistical Society, Series B (Methodological) 39(1):1-38.

Downs, A. 1957. An economic theory of democracy. New York: Harper \& Row.

Eddelbuettel, D., and R. François. 2011. Rcpp: Seamless R and C++ integration. Journal of Statistical Software 40(8):1-18.

Elff, M. 2009. Social divisions, party positions, and electoral behaviour. Electoral Studies 28(2):297-308.

2012. Replication data for: A dynamic state-space model of coded political texts. http://hdl.handle.net/1902.1/19198 (accessed December 11, 2012) IQSS Dataverse Network [Distributor] V1 [Version].

Enelow, J. M., and M. Hinich. 1984. The spatial theory of voting: An introduction. Cambridge, MA: Cambridge University Press.

. 1990. Advances in the spatial theory of voting. Cambridge, MA: Cambridge University Press.

Evans, G., A. Heath, and C. Payne. 1999. Class: Labour as a catch-all party? In Critical elections: British parties and voters in long-term perspective, eds. G. Evans and P. Norris. London; Thousand Oakes, CA; New Delhi: Sage Publications.

Ezrow, L. 2007. The variance matters: How party systems represent the preferences of voters. Journal of Politics 69(1):182-92.

Fielding, S. 2003. The Labour party: Continuity and change in the making of "New" Labour. New York: Palgrave Macmillan.

Francois, R., D. Eddelbuettel, and D. Bates. 2011. RcppArmadillo: Rcpp integration for Armadillo templated linear algebra library. $\mathrm{R}$ package version 0.2.18. http://cran.r-project.org/web/packages/RcppArmadillo/. (accessed December 23, 2012).

Gentle, J. E. 2003. Random number generation and Monte Carlo methods. 2nd ed. New York: Springer.

Heath, A., R. Jowell, J. Curtice, G. Evans, J. Field, and S. Witherspoon. 1991. Understanding political change: The British voter 1964-1987. Oxford: Pergamon Press.

Hinich, M. J., and M. C. Munger. 1994. Ideology and the theory of political choice. Ann Arbor: University of Michigan Press.

Klingemann, H.-D., A. Volkens, J. Bara, I. Budge, and M. McDonald. 2006. Mapping policy preferences II. Estimates for parties, electors, and governments in Eastern Europe, the European Union, and the OECD, 1990-2003. Oxford: Oxford University Press.

Kollman, K., J. H. Miller, and S. E. Page. 1992a. Adaptive parties in spatial elections. American Political Science Review 86(4):929-37.

- 1992b. Political parties and electoral landscapes. British Journal of Political Science 28(1):139-58.

Laver, M. 2001. Position and salience in the policies of political actors. In Estimating the policy positions of political actors, ed. M. Laver, 66-75. London/New York: Routledge.

Laver, M. J., and J. Garry. 2000. Estimating policy positions from political texts. American Journal of Political Science 44(3):619-34.

Laver, M., K. Benoit, and J. Garry. 2003. Extracting policy positions from political texts using words as data. American Political Science Review 97(2):311-31.

Little, R. J., and D. B. Rubin. 2002. Statistical analysis with missing data. 2nd ed. Hoboken, NJ: Wiley.

Lowe, W., K. Benoit, S. Mikhaylov, and M. Laver. 2011. Scaling policy preferences from coded political texts. Legislative Studies Quarterly 34(1):123-55. 
Martin, A. D., and K. M. Quinn. 2002. Dynamic ideal point estimation via Markov chain Monte Carlo for the U.S. Supreme Court, 1953-1999. Political Analysis 10(2):134-53.

McDonald, R. P. 1999. Test theory: A unified treatment. Mahwah, NJ; London: Lawrence Erlbaum.

McKelvey, R. 1979. General conditions for global intransitivities in formal voting models. Econometrica 47(5):1085-112.

Mikhaylov, S., M. Laver, and K. R. Benoit. 2012. Coder reliability and misclassification in the human coding of party manifestos. Political Analysis 20(1):78-91.

Poole, K. T., and H. Rosenthal. 1985. A spatial model for legislative roll call analysis. American Journal of Political Science 29(2):357-84.

R Development Core Team. 2011. R: A Language and Environment for Statistical Computing. Vienna, Austria: R Foundation for Statistical Computing.

Ripley, B. D. 1987. Stochastic simulation. Hoboken, NJ: Wiley.

Robert, C. P., and G. Casella. 2004. Monte Carlo statistical methods. 2nd ed. New York: Springer.

Slapin, J. B., and S.-O. Proksch. 2008. A scaling model for estimating time-series party positions from texts. American Journal of Political Science 52(3):705-22.

Stegmueller, D. 2011. Apples and oranges? The problem of equivalence in comparative research. Political Analysis 19(4):471-87.

Volkens, A., O. Lacewell, S. Regel, H. Schultze, and A. Werner. 2010. The Manifesto Data Collection. Berlin: Wissenschaftszentrum Berlin für Sozialforschung (WZB). https://manifestoproject.wzb.eu/ (accessed December 11, 2012).

Weakliem, D. L., and A. F. Heath. 1999. The secret life of class voting: Britain, France, and the United States since the 1930s. In The end of class politics? Class voting in comparative context, ed. G. Evans, 97-136. Oxford: Oxford University Press.

Wei, G. C., and M. A. Tanner. 1990. A Monte Carlo implementation of the EM algorithm and the poor man's data augmentation algorithms. Journal of the American Statistical Association 85(411):699-704. 